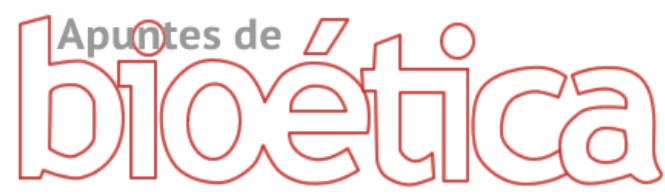

https://doi.org/10.35383/apuntes.v2i1.238

\title{
CRISPR/Cas9: Edición Genética vs. Bioética
}

Un análisis bioético de la técnica de edición de genes llamada CRISPR/Cas9

\author{
Mirielle Liliana Palacios Yabar ${ }^{1}$
}

\section{INFORMACIÓN DEL ARTÍCULO RESUMEN}

Recibido el 19 de marzo de 2019

Aceptado el 08 de julio de 2019

\section{Palabras claves:}

CRISPR/Cas9

Bioética

Edición genética

Biotecnología

Investigación biomédica

Desde el descubrimiento de la doble hélice por Watson y Crick, los avances biotecnológicos en relación al mundo de la genética, han dado rápidos saltos para la humanidad. Grandes hitos como el secuenciamiento del ADN, el Proyecto del Genoma Humano, la clonación de la oveja Dolly, e incluso el desarrollo de gatos fosforescentes modificados genéticamente para brillar en la oscuridad, entre otros, han sido posibles con estos avances. La ingeniería genética es una posibilidad gracias a este desarrollo de la ciencia, y es así que desde el año 2012, la aparición de una nueva técnica que utiliza el sistema conocido como CRISPR/Cas9 ha abierto las fronteras a nivel global, a la era de la edición de genes, de una manera más rápida, fácil y accesible. Al hablar de nuevas tecnologías de modificación genética, especialmente si se trata de la modificación de la línea germinal, es importante tener en cuenta el impacto bioético, que trataremos en el presente artículo.

\section{CRISPR/Cas9: Gene Editing vs. Bioethics}

A bioethical analysis of the gene editing technique called CRISPR/Cas9

\section{ABSTRACT}

\section{Keywords:}

CRISPR/Cas9

Bioethics

Gene editing

Biotechnology

Medical research
Since the discovery of the double helix by Watson and Crick, biotechnological advances related to the world of genetics, have yielded rapid jumps for humanity. Major milestones such as DNA sequencing, the Human Genome Project, the cloning of the Dolly sheep, and even the development of genetically modified phosphorescent cats to shine in the dark, among others, have been possible with these advances. Genetic engineering is a possibility thanks to this development of science, and so

${ }^{1}$ Licenciada en Farmacia y Bioquímica en la Universidad Peruana Cayetano Heredia. Diplomada en Inmunología Básica y Clínica en la Universidad Peruana Cayetano Heredia. Diplomada en Bioética en la Investigación Biomédica y Clínica en la Universidad Católica Santo Toribio de Mogrovejo. Analista de Farmacovigilancia. Email: mirielle.palacios@gmail.com. ORCID ID: https://orcid.org/0000-0001-9614-6036 
since 2012, the emergence of a new technique that uses the system known as CRISPR/Cas9 has opened the borders globally, to the era of gene editing, in a faster, easier and more accessible way. When talking about new technologies of genetic modification, especially if it is about the modification of the germ line, the bioethical impact must be taken into account, which will be discussed in the present article.

\section{Introducción}

La edición genética ya es posible en la actualidad con el desarrollo científico que ha logrado avanzar rápidamente a través de los años desde el descubrimiento de la doble hélice del ADN por Watson y Crick.

Actualmente, para la investigación de edición genética específica se conocen cuatro métodos, dentro de las cuales tenemos la ZFN (por sus siglas en inglés: Zinc Finger Nucleases), TALEN (por sus siglas en inglés: Transcription Activator-Like Effector based Nucleases), Meganucleases, y el sistema CRISPR/Cas9 (por sus siglas en inglés: Clustered Regions of Interspersed Palindromic Repeats-Cas9) (Riordan, Heruth, Zhang, \& Ye, 2015, pág. 1). Esta última, es la más popular y más usada por sus ventajas, por lo que se usa cada vez más en investigaciones $\mathrm{y}$ hay un incremento en sus publicaciones a través de los años (Gómez-Tatay \& Aznar, 2019, págs. 173-174).

El sistema CRISPR/Cas9 como se conoce actualmente, fue utilizado por primera vez el 2012 por el equipo liderado por Doudna y Charpentier para cortar ADN in vitro, quienes descubrieron que Cas9 era una endonucleasa de ADN programable por ARN, lo que dio lugar a una serie de publicaciones después de este descubrimiento (Doudna \& Charpentier, 2014, págs. 1258096-2-1258096-2). Es así que en el año 2013, el equipo de Feng Zhang, usó CRISPR/Cas9 para cortar el genoma de las células de mamíferos (Cong, et al. 2013, pág. 819). Sin embargo, este sistema CRISPR fue descrito por primera vez por científicos japoneses en 1987 como secuencias repetidas en el genoma de E.coli (Doudna \& Charpentier, 2014, págs. 1258096-2-1258096-3). En el 2005 se descubrió que CRISPR formaba parte de un sistema de defensa viral (Gómez-Tatay \& Aznar, 2019, pág. 173) encontrado en bacterias y arqueas (Riordan, Heruth, Zhang, \& Ye, 2015, pág. 1).

Este método CRISPR/Cas9 se basa en el mecanismo natural de reparación de cortes que se dan en las cadenas del ADN, es por eso que consiste en la utilización de dos componentes principales, Cas9 que es la enzima que corta el ADN como si fueran unas tijeras y una pequeña secuencia de ARN guía (también llamada CRISPR ARN) la cual indica a la enzima Cas9 el lugar donde debe cortar (Ledford, 2016, págs. 157158). Cuando ocurre este corte llamado DSB (por sus siglas en inglés: double stranded break), las células se reparan naturalmente mediante dos mecanismos conocidos como el HDR (por sus siglas en inglés: homologa directed repair) o por NHEIJ (por sus siglas en inglés: non-homologous end joining), esto hace que se inserten, borren o muten ciertas secuencias de ADN para reparar el corte (Riordan, Heruth, Zhang, \& Ye, 2015, págs. $1-2)$.

Actualmente esta tecnología CRISPR/Cas9, como mencionan los autores Gómez-Tatay y Aznar: "ha permitido la modificación eficiente y especifica del genoma en muchas especies" (Gómez-Tatay \& Aznar, 2019, pág. 174), lo cual ha sido útil en ingeniería genética como una mejora en la creación de animales transgénicos 
para investigación biomédica y en agricultura, incluyendo ratones, ratas, Drosophila melanogaster, Caenorhabditis elegans, Saccharomyces cerevisiae y Dani rerio, como modelos tradicionales. $Y$ en cerdos y cabras como modelos no tradicionales. Además, se sabe también que se ha usado para crear modelos primate de enfermedades para investigación. (Riordan, Heruth, Zhang, \& Ye, 2015, pág. 7).

El uso del sistema CRISP/Cas9 como una herramienta de edición genética es de fácil y rápido manejo, y es preciso y accesible en costo a diferencia de los otros métodos, que puede ser usado por una persona con conocimientos básicos de técnicas en genética. Estas razones la convierten en una herramienta muy poderosa, con un futuro muy prometedor y esperanzador para ayudarnos a entender, prevenir y curar muchas enfermedades del ser humano, especialmente si se puede editar la línea germinal. Sin embargo, se tienen muchas interrogantes tanto científicas como bioéticas para decidir si finalmente es una buena opción.

El comité de las Academias Nacionales de Estados Unidos (de ciencia, ingeniería y medicina), indican que no se debe editar el genoma humano más que con fines de tratamiento o prevención de enfermedades y discapacidades (Dance, 2017, pág. 1009). Además, la Convención de Oviedo, en su artículo 13, permite la edición del genoma sólo con fines de prevención, diagnóstico de enfermedades y para terapias, pero prohíbe las ediciones genéticas en las líneas germinales que puedan pasarse a otras generaciones (Council of Europe, 1997).

Sin embargo, a pesar de ciertos lineamientos escasos que puedan existir hasta el momento, y ante el avance acelerado de estas técnicas de edición genética, existe un caso por el momento de edición genética en embriones que han sido editados genéticamente y fueron llevados a término, a pesar de los peligros y riesgos que conlleva el usar esta técnica que aún no ha sido perfeccionada.

Es por este motivo y por el impacto que esta técnica pueda tener en la sociedad y en futuras generaciones, que es importante realizar un análisis bioético del tema poniendo como centro la dignidad del ser humano, con el objetivo de dar luces y recordar que la técnica debe servir al hombre y buscar su beneficio y no dañar su integridad, y que pueden existir consecuencias para el ser humano cuando se toma muy a la ligera el uso de este tipo de herramientas.

\section{Metodología}

La presente investigación tiene un enfoque cualitativo (Hernandez Sampieri, 2014, págs. 219) de tipo documental (Bernal, 2016, págs. 110-120) basada en la búsqueda bibliográfica en bases de datos de artículos científicos y ensayos clínicos relacionados a la técnica de edición genética CRISPR/Cas9 desde su descubrimiento hasta la actualidad con el objetivo de estudiar el avance de esta técnica a través del tiempo, conocer la realidad de su uso en la actualidad y analizar las repercusiones bioéticas que puedan tener en el futuro.

\section{Método de búsqueda}

Se realizó la búsqueda bibliográfica para la obtención de artículos científicos relacionados a la técnica CRISPR/Cas9 dentro de los repositorios virtuales MEDLINE (PUBMED), Science Direct, Nature, Science y Google Scholar, poniendo como único límite el idioma de la publicación en inglés o en español. Asimismo, se buscaron ensayos clínicos actualmente registrados dentro del repositorio global ClinicalTrials.gov y 
localmente en el Registro Peruano de Ensayos Clínicos (REPEC) con las mismas especificaciones.

Adicionalmente, se realizó la búsqueda de artículos relacionados al tema en bases de datos de bioética como Persona y Bioética, revista de la Universidad de la Sabana y Cuadernos de Bioética, revista de la Asociación Española de Bioética y Ética Médica.

Para la búsqueda de las referencias bibliográficas en los repositorios de artículos científicos, se usaron las palabras "bioethics", "CRISPR/Cas9" "CRISPR-Cas9", "gene editing", "genome editing", "biomedical discoveries" con su correspondiente traducción en español "bioética", "CRISPR/Cas9" "CRISPR-Cas9", "edición de genes", "edición genética", "descubrimientos biomédicos".

Se emplearon los operadores booleanos AND y OR (Centro Cochrane Iberoamericano, 2012, pp. 149-150) para la realización de la búsqueda (CRISPR/Cas9 OR CRISPR-Cas9) AND (gene editing OR genome editing) AND bioethics AND "biomedical discoveries"

Se seleccionaron los artículos que tuvieran como objetivo explicar la utilidad y aplicaciones de la técnica CRISPR/Cas9 en la actualidad, historia y definiciones científicas, aspectos bioéticos de la técnica y su uso en humanos. Y se excluyeron todas aquellas referencias provenientes de literatura gris, que no sean de acceso abierto y que el texto completo no se encuentre disponible.

\section{Resultados, análisis y discusión}

Dentro de las bases de datos para ensayos clínicos ClinicalTrials.gov y REPEC se obtuvieron los siguientes resultados (Tabla 1):
Tabla 1. Ensayos Clínicos Registrados para CRISPR

CLINICAL TRIALS (A nivel mundial)

26 Registros

REPEC (En Perú)

0 Registros

A nivel mundial existen 26 ensayos clínicos en total usando la tecnología CRISPR. La mayoría de los estudios clínicos registrados actualmente en ClinicalTrials.gov, son desarrollados en China (12) y en Estados Unidos (8). En Perú no existe ningún ensayo clínico registrado usando esta tecnología (Tabla 2).

\begin{tabular}{lc} 
Tabla 2. Estudios Clínicos Registrados en Clinical Trials para CRISPR por país \\
\hline País & Estudio Clínico \\
\hline China & 12 \\
Erancia & 1 \\
Gran Bretaña & 1 \\
Desconecida & 3 \\
Estados Unidos & 8 \\
Estados Unidos, Alemania, Italia (Multipais) & 1 \\
\hline Total & $\mathbf{2 6}$
\end{tabular}

De los 26 ensayos clínicos encontrados en total a nivel mundial en ClinicalTrials.gov, encontramos que 14 de ellos se encuentran a la fecha en enrolamiento, 1 fue suspendido, 1 retirado, 2 activos sin enrolamiento, 3 sin iniciar enrolamiento y 1 en estatus desconocido (Tabla $3)$.

\begin{tabular}{lc} 
Tabla 3. Estatus de Estudios Clinicos Registrados en Clinical Trials para CRISPR \\
\hline Estatus & Estudios Clínicos \\
\hline Active, sin enrolamiente & 2 \\
Completade & 1 \\
Sin iniciar enrolamiento & 3 \\
En enrolamiente & 14 \\
Suspendide & 1 \\
Estatus desconocide & 1 \\
Retirado & 4 \\
\hline Total & $\mathbf{2 6}$
\end{tabular}

Adicionalmente, sólo 1 estudio desarrollado en China ha sido completado, el cual tenía el objetivo de estudiar la seguridad de 
un tratamiento con infusión de células autólogas modificadas genéticamente con la tecnología CRISPR/Cas9 en pacientes con cáncer de esófago avanzado (Tabla 4)

\begin{tabular}{lc} 
Tabla 4. Estatus de Estudios Clinicos Registrados en Clinical Trials para CRISPR por país \\
\hline Estatus por pais & Estudio Clínico \\
\hline China & $\mathbf{1 2}$ \\
\hline Active, sin enrolamiente & 1 \\
\hline Completado & 1 \\
\hline En enrolamiente & 7 \\
\hline Estatus desconocide & 1 \\
\hline Retirade & 2 \\
\hline Erancia & $\mathbf{1}$ \\
\hline Active, sin enrolamiente & 1 \\
\hline Gran Bretaña & $\mathbf{1}$ \\
\hline En enrolamiente & 1 \\
\hline Desconocide & $\mathbf{3}$ \\
\hline Sin iniciar enrolamiente & 1 \\
\hline Retirade & 2 \\
\hline Estados Unidos & $\mathbf{8}$ \\
\hline Sin iniciar enrolamiente & 2 \\
\hline En enrolamiente & 5 \\
\hline Suspendide & 1 \\
\hline Estados Unidos, Alemania, Italia & $\mathbf{1}$ \\
\hline En enrolamiente & 1 \\
\hline Total & $\mathbf{2 6}$ \\
\hline
\end{tabular}

Sin embargo, hasta la fecha no se han publicado resultados de ninguno de los ensayos registrados (Tabla 5).

\begin{tabular}{lc} 
Tabla 5. Resultados de Estudios Clínicos Registrados en Clinical Trials para CRISPR por país \\
\hline Resultados publicados por país & Estudio Clínico \\
\hline China & $\mathbf{1 2}$ \\
\hline Resultados no publicados & 12 \\
Erancia & $\mathbf{1}$ \\
\hline Resultados no publicados & 1 \\
Gran Bretaña & $\mathbf{1}$ \\
\hline Resultados, no publicados & 1 \\
Desconocide & $\mathbf{3}$ \\
\hline Resultados no publicados & 3 \\
Estados Unidos & $\mathbf{8}$ \\
\hline Resultados, no publicados & 8 \\
Estados Unidos, Alemania, Italia & $\mathbf{1}$ \\
\hline Resultados no publicados & 1 \\
\hline Total & $\mathbf{2 6}$
\end{tabular}

Reporte de casos de uso de la técnica CRISPR/Cas9 en humanos:

\section{Caso de China}

En noviembre del 2018, un profesor asociado de la Universidad del Sur de Ciencia y Tecnología de Shenzhen en China, Ilamado He Jiankui, indicó en una cumbre en Hong Kong, haber editado genéticamente embriones humanos con la técnica de CRISPR/Cas9 (Li, Walker, Nie, \& Zhang, 2019, pág. 33), a los cuales, según Sheldon Krimsky (Krimsky, 2019, págs. 19-20), llevó a término por medio de una fertilización in vitro de la cual nacieron gemelas. Con esta acción, violó el consenso internacional que existe desde la cumbre de edición genética humana del 2015, en el que se cuestionó la edición genética de la línea germinal hasta que se resuelvan los problemas de seguridad y eficacia que conlleva la aplicación de esta nueva técnica.

Se añade a su lista de transgresiones, el quebrar las guías éticas que existen en China para investigación con embriones por lo que este estudio clínico fue retirado del Registro de Ensayos Clínicos de China, y además quebró la normativa ética de la misma universidad para la que trabajaba, por lo que no obtuvo la aprobación ética para desarrollar la investigación. Por su parte, publicó sus propias guías éticas como justificación para su ensayo clínico, las cuales él mismo no cumplió. Se conoce también que no reportó estudios previos de edición con CRISPR en embriones de ratones, primates o humanos, y el reporte que presentó de mutaciones "off-target", que son usuales cuando se usa el sistema CRISPR/Cas9 y que puede ser peligroso para el ser humano, fue insuficiente. Adicionalmente a esto, el consentimiento informado no fue aceptable ya que no informaba claramente a los padres acerca del estudio clínico en el que iban a participar y no declaró conflictos de intereses (Krimsky, 2019, págs. 19-20) (Li, Walker, Nie, \& Zhang, 2019, págs. 33-36). 


\section{Caso de Rusia}

A menos de un año después de la cumbre de noviembre del 2018, donde el científico chino He Jiankui mostró su investigación sobre la aplicación del sistema CRISPR/Cas9 en humanos al mundo, un biólogo ruso Denis Rebrikov anunció que estaba considerando implantar embriones modificados genéticamente en mujeres a finales de este año si obtiene la aprobación, ya que plantea que su técnica tiene más beneficios, menos riesgos y sería más aceptable a nivel ético que la investigación de He Jiankui. (Cyranoski, 2019, pág. 145)

Este anuncio causó ciertas reacciones tanto de científicos como bioeticistas, quienes demostraron preocupación al creer que aún se necesitan más pruebas antes de empezar a usar este tipo de tecnología para alterar genes en los seres humanos ya que puede afectar a las generaciones futuras. (Cyranoski, 2019, pág. 146)

\section{Análisis bioético}

La edición genética es aún experimental y está asociada a mutaciones off-target según Julian Savulescu, un eticista de la Universidad de Oxford (Normile, 2018). Lo cierto es, que no se conoce en su totalidad los riesgos o consecuencias que puedan existir especialmente cuando se editan genes de las líneas germinales, lo cual puede afectar a todas las generaciones y no solo a la persona con la que se experimenta. Como mencionan los autores Jin-ru Li, et. al ( $\mathrm{Li}$, Walker, Nie, \& Zhang, 2019, pág. 35), los bebés que hayan sido editados genéticamente correrán el riesgo de sufrir efectos secundarios por las mutaciones "off target" que son inevitables porque esta nueva tecnología es limitada.

Si bien la técnica del sistema CRISPR/Cas9 es fácil de usar ya que se requiere sólo un conocimiento básico de manipulación genética, es accesible por su bajo costo a comparación de las otras tecnologías, y es más específica y precisa para cambios en puntos específicos del genoma (De Lecuona, Casado, Marfany, Lopez Baroni, \& Escarrabill, 2017, pág. 675), es importante analizar detenidamente las repercusiones bioéticas que puede traer consigo esta técnica.

Se sabe que esta técnica CRISPR/Cas9, se usa no sólo para investigación dentro del campo médico, sino también en el campo de la agricultura y ganadería, lo cual podría ayudar a la humanidad a resolver el problema de la falta de alimentos o incluso evitar la transmisión de enfermedades por medio de vectores. Sin embargo, se debe tener en cuenta que también puede llevar a la extinción de especies (GamboaBernal, 2016, pág. 127). Un ejemplo de esto es el estudio de una población en cautiverio de mosquitos transmisores de malaria, Anopheles gambiae, en la que mediante una modificación genética con el sistema CRISPR/Cas9, se logró suprimir la capacidad reproductiva de la población en las generaciones 8 y 12 las cuales no produjeron huevos y por consiguiente la extinición de la especie (Kyrou, et al., 2018, págs. 1064-1065). Estos resultados generan grandes interrogantes ante el uso de esta técnica, ya que no se tienen claras las consecuencias que podrían traer consigo.

Según los autores Gómez-Tatay y Aznar (Gómez-Tatay \& Aznar, 2019, pág. 176), ya se han publicado artículos científicos en los que se ha aplicado la técnica CRISPR en la línea germinal, y los primeros estudios fueron realizados en China sobre embriones sobrantes de fertilizaciones in vitro. Investigaciones como estas, con embriones viables, se han realizado en países como Inglaterra, Estados Unidos y Suecia. Adicionalmente, mencionan que en uno de los trabajos producían embriones mediante clonación. 
Ante lo presentado en China por $\mathrm{He}$ Jiankui, la comunidad científica y el público en general respondió con críticas y preocupación ante esta controversia (Cribbs \& Perera, 2017, pág. 628), empezando por científicos chinos y divisiones de la Academia de Ciencia de China, quienes se opusieron a la edición del genoma en embriones con esta técnica por poseer muchos riesgos de mutaciones "off target" que no se pueden controlar, y ya que regulaciones $y$ principios éticos prohíben estas aplicaciones (Li, Walker, Nie, \& Zhang, 2019, pág. 33).

Adicionalmente, el Instituto Nacional de Salud de Estados Unidos (NIH: por sus siglas en inglés National Institutes of Health) en base a este hecho, declaró no apoyar el uso de tecnologías de edición genética en embriones humanos y que la aplicación de la técnica para edición humana de la línea germinal en este caso en particular se ha realizado muy irresponsablemente, por lo que establece que es necesario el desarrollo de un consenso internacional que establezca límites para este tipo de investigaciones (Collins, 2018).

Asimismo, el 2019 la Organización Mundial de la Salud, indicó su posición sobre las ediciones genéticas en humanos sobre líneas germinales concluyendo que actualmente sería irresponsable hacer ediciones que podrían afectar a las generaciones (Ledford, 2019, pág. 296).

El Observatorio de Bioética y Leyes (OBD) (por sus siglas en catalán: Observatori de Bioètica i Dret) de la Universidad de Barcelona, también fue uno de los primeros en dar declaraciones al respecto, señalando que se debe analizar esta técnica "desde una perspectiva multidisciplinaria, abriendo las puertas al debate social y promover una investigación éticamente aceptable" (De Lecuona, Casado, Marfany, Lopez Baroni, \& Escarrabill, 2017, pág. 680). Esta apertura al diálogo social es importante ya que se habla de consecuencias graves no solo para la salud o bienestar de un solo individuo, sino para toda la sociedad (Ledford, 2019, pág. 294).

La Academia Nacional de Ciencias de Estados Unidos (NAS: por sus siglas en inglés National Academy of Sciences) y la Academia Nacional de Medicina (NAM: por sus siglas en inglés National Academy of Medicine) indican la importancia del compromiso público en este debate de la edición genética "para que exista una relación saludable entre la ciencia y la sociedad" y se debe considerar los beneficios y riesgos que existe en la aplicación de estas técnicas de edición genética (Cribbs \& Perera, 2017, pág. 629).

Tanto el compromiso público como el científico y a nivel de los gobiernos es necesario en este debate para buscar la forma de disuadir a los científicos a experimentar con la edición de genes de la línea germinal ("Act now on CRISPR babies", 2019), esto es muy importante ya que así como el caso de la propuesta controversial del científico ruso Denis Rebrikov, que pretende seguir las huellas del científico chino He Jianku, cualquier otro científico en el mundo puede realizar los mismos experimentos, especialmente en aquellos países donde no hay la debida regulación.

Actualmente existe un moratorio en cuanto a la edición genética de la línea germinal en muchos países de Europa, en Estados Unidos y en China (Cribbs \& Perera, 2017, pág. 629), y científicos concuerdan en la necesidad de regulaciones, leyes y guías tanto éticas como técnicas para estandarizar la investigación de esta técnica y su aplicación (Li, Walker, Nie, \& Zhang, 2019, pág. 37).

Para esto, llaman a buscar un consenso global para regular esta técnica tanto por medio de leyes como guías éticas a seguir ya que el mal 
uso de esta técnica puede tener un impacto en el futuro de generaciones en toda la humanidad. (De Lecuona, Casado, Marfany, Lopez Baroni, \& Escarrabill, 2017, pág. 680).

Existen diferentes opiniones al respecto, según los autores De Lecuona, et al, prohibir la aplicación de esta técnica no es suficiente, para ellos es necesario "pensar a nivel nacional e internacional como regular esta técnica que ha cambiado la perspectiva bioetica y legal que se ha tenido hasta ahora por ejemplo de la naturaleza del embrión o de las células madres embrionarias y sus aplicaciones" (De Lecuona, Casado, Marfany, Lopez Baroni, \& Escarrabill, 2017, pág. 680).

Sin embargo, para Gamboa-Bernal si es importante mantener la prohibición de no aplicar esta técnica en la línea germinal humana y que la investigación en líneas somáticas "no se realice a costa de la vida de los embriones" (Gamboa-Bernal, 2016, pág. 128).

Según Gamboa-Bernal (Gamboa-Bernal, 2016, pág. 126), no se descarta el riesgo de ediciones incorrectas y mutaciones no deseadas que puedan generar defectos genéticos, y es de mucho más preocupación si son generadas en la línea germinal por afectar también a generaciones futuras. En estos casos, como menciona el bioeticista Bryan Cwik, existe el cuestionamiento de cómo deberían monitorear los científicos a las generaciones de aquellas personas modificadas genéticamente para poder buscar efectos transgeneracionales (Ledford, 2019, pág. 296).

Tampoco se descarta el riesgo de discriminación e incluso la eugenesia, ya que no existe una línea bien definida entre la aplicación de CRISPR/Cas9 para terapia y lo que se llama "enhancement" o mejoramiento (De Lecuona, Casado, Marfany, Lopez Baroni, \& Escarrabill,
2017, pág. 675) (Cribbs \& Perera, 2017, pág. 629).

Con esta técnica, el "mejoramiento genético" no sería imposible y podría usarse no sólo en humanos, sino también en la agricultura y en los animales. $Y$ si bien cambios genéticos en animales y en la agricultura puedan ser de preocupación al no saber qué repercusiones puedan tener para con el ser humano y para el ecosistema, si nos centramos en el mejoramiento del ser humano, se abre la puerta a la eugenesia, con la cual, según los autores Bermeo-Antur y Quimbaya, "...la descendencia no sería fruto de lo casual, por el contrario, sería el efecto de acciones pensadas, medidas y calculadas para poder eliminar las anomalías genéticas que podrían causar defectos físicos visibles." (Bermeo-Antury \& Quimbaya, 2016, pág. 208), creando así una raza humana superior $y$ diferenciada genéticamente de los que no pasen por este proceso.

La línea entre tratamiento y mejoramiento genético aún no está bien dividida, por lo que existen muchos cuestionamientos ante lo que se debería o no hacer (Dance, 2017, pág. 1009) y si la sociedad estaría lista para este tipo de tecnologías (Dance, 2017, pág. 1010) (Ledford, 2019, pág. 295).

Según Nature, una encuesta en el Reino Unido encontró que el $83 \%$ de los participantes estaba de acuerdo con modificar genéticamente la línea germinal para tratar enfermedades que no tenían cura, pero $60 \%$ se opusieron a la edición genética con el fin de mejorar la inteligencia por ejemplo (Ledford, 2019, pág. 296).

Asimismo, en Japón se realizó también una encuesta en la que se obtuvo que la mayorīa generalmente aceptó el uso de la edición genética para enfermedades pero muchos demostraron preocupación en cuanto a los 
riesgos (Uchiyama, Nagai, \& Muto, 2018, págs. 745-748).

Es importante recordar también que las investigaciones realizadas actualmente con la técnica CRISPR/Cas9 para edición genética humana sobre las líneas germinales se desarrollan generalmente sobre embriones humanos no viables, a los cuales no pueden llevar a término por razones legales y éticas. Además, se sabe por la literatura científica que al menos la mitad de los 10 embarazos en los experimentos con monos terminaron en abortos espontáneos. (Vogel, 2015, pág. 1301)

Según la revista Science un consenso del Hinxton Group (un grupo con miembros de Canada, Reino Unido, Estados Unidos, Italia, Alemania, Mexico, Israel y Países Bajos), del 2015 , indica que es necesario que se prueben este tipo de tecnologías de edición de genes en embriones humanos, para determinar si pueden ser seguras y eficaces, por lo que promueve a los científicos a "considerar cuidadosamente la categoría de embrión utilizado", mencionan que los que provienen de fertilizaciones in-vitro puedan no aportar buenos resultados, y que ciertos experimentos requerirán que los investigadores creen nuevos embriones específicos para la investigación que realicen (Vogel, 2015). El autor Gamboa-Bernal manifiesta que 'aunque se tengan grandes posibilidades de éxito en términos de curación de enfermedades o de mejoramientos de la condición humana, siempre será descalificada la técnica si en su desarrollo mueren seres humanos, así estén solo en estado embrionario." (Gamboa-Bernal, 2016, pág. 125).

Tras el escándalo del caso del investigador de China, algunos científicos se han puesto en alerta por el daño que este tipo de noticias pueda causar al mundo científico. Su mayor preocupación es que, al haberse realizado esta investigación sin seguir los principios éticos establecidos en consenso hasta la actualidad y al no considerar el riesgo innecesario que se pueda causar a los sujetos de investigación (Normile, 2018), se puede generar un estigma en la sociedad y forzar a los científicos a frenar los avances de la ciencia (Gómez-Tatay \& Aznar, 2019, pág. 176).

Por estos comentarios, se podría observar que algunos científicos se enfocan más en evitar que la sociedad proteste y frene el avance científico en relación a la edición genética con CRISP/Cas9, antes que resguardar la dignidad humana y la integridad física de los sujetos de investigación.

Según Elio Sgreccia, para valorar este tipo de intervenciones, se debe tener en cuenta el principio terapéutico, que se encuentra estrechamente relacionado con el respeto de la integridad física de la persona, que incluye su genoma (Sgreccia, 2014, pág. 421). Es entonces que, la terapia génica, en este caso la edición genética con el uso del sistema CRISPR/Cas9 en la línea germinal y somática sólo en casos terapéuticos, sería lícita cuando "no suprima embriones humanos, no determine riesgos graves de error y aberraciones peores que las que se quieren corregir o que tenga alguna posibilidad de éxito" (Sgreccia, 2014, pág. 928). Sin embargo, la edición genética de la línea germinal terapéutica no sería aceptable por dos razones: cuando el modelo usado no alcance el resultado terapéutico y haya riesgos difíciles de controlar, así como cuando se busque modificar el genoma sobrepasando el objetivo terapéutico (Sgreccia, 2014, pág. 421). Es decir, el sistema CRISPR/Cas9 en líneas germinales para fines terapéuticos son ilícitos debido a que en la actualidad aún no se conoce la solución a los riesgos y mutaciones que existen y que aún no son controlables. $Y$ en el caso de su uso para mejoramiento o "enhancement" la ilicitud es absoluta. 


\section{Conclusiones}

La técnica de edición genética usando CRISP/Cas9 ha hecho más accesible, reducido el costo y aumentado el interés de los científicos para hacer investigación en genética, lo que puede llevar a un conocimiento más profundo de los genes y su relación con las enfermedades, y así lograr el descubrimiento de la cura para las mismas.

Sin embargo, se debe tener en consideración que existen muchos interrogantes y áreas desconocidas de esta técnica y su aplicación, que causan preocupación en científicos, en bioeticistas, y en el público en general por sus repercusiones legales, éticas y morales.

Dentro de estas repercusiones podemos observar claramente que el uso irresponsable de esta técnica en humanos es muy factible, ya que al ser por el momento un territorio no tan explorado, no se conoce en su totalidad lo que pueda causar a la larga una pequeña deleción o un pequeño cambio dentro del ADN.

Un ejemplo de esto es la investigación muy criticada de He con la técnica CRISPR/Cas9 en embriones humanos, la cual puede ser considerada más como un experimento que como un ensayo clínico, por haberse realizado sin tomar las debidas precauciones de seguridad correspondientes a esta técnica, ni haberse evaluado el riesgo y beneficio para con los sujetos de investigación, y por quebrar normas legales y éticas, que lo llevaron a no contar con una aprobación por un comité de ética institucional y no tener el respaldo por el instituto de salud del país. Esto conlleva grandes repercusiones ante la sociedad y el mundo científico, ya que será muy difícil conocer los resultados de lo que pase con las gemelas en un futuro.
Adicionalmente a eso, lo que más preocupa a los bioeticistas es el uso eugenésico que se le podría dar a esta técnica, ya que al ser accesible y más fácil de usar, se puede crear bebés de acuerdo a la conveniencia y pedido de los padres, llevando incluso a la eliminación de algún rasgo físico por motivos de gusto mas no de enfermedad o riesgo. Es por esto que nos debemos preguntar sobre la intención del uso de la técnica, si es para uso terapéutico o si es para uso eugenésico y tener bien en claro las motivaciones por existir una línea muy delgada entre estas.

Será un desafío muy grande para los científicos en los años siguientes, el seguir investigando formas de hacer que esta técnica con el sistema CRISPR/Cas9 sea más segura y mejor controlada, que se puedan obtener resultados esperados para su uso clínico, especialmente si se edita la línea germinal, ya que deberá ser medido a través de las generaciones, y esto último también genera un cuestionamiento de cómo hacerlo.

Actualmente, aún no existen resultados publicados de los ensayos clínicos que se están llevando a cabo por el momento, lo que quiere decir que queda un largo camino por recorrer con esta técnica y con todo lo nuevo que pueda generarse en el futuro. Por lo que es importante que se siga muy de cerca el desarrollo en especial de esta técnica CRISPR/Cas9 para evitar que se sigan usando a costa de la vida y bienestar de las personas.

Está previsto que para el año 2020, un comité de la comunidad internacional científica, liderada por las Academias Nacionales de Ciencia y Medicina de Estados Unidos junto con la Royal Society del Reino Unido, publique un reporte final acerca de las investigaciones que realicen acerca de los problemas científicos, éticos y sociales relacionados a la edición genética.

Actualmente se discute que exista un consenso global de lo aceptable dentro de la edición 
genética con CRIPSR/Cas9 y con las nuevas tecnologías que se desarrollen en el futuro. Sin embargo, llegar a este consenso será muy complicado por el diferente entendimiento que se tiene sobre conceptos básicos como embrión, persona, calidad de vida o dignidad humana. Es por eso, que lo crucial a la hora de establecer lineamientos o hacer leyes, es tener en cuenta y poner por sobre todo la dignidad humana en su totalidad, de lo contrario, se podría poner en riesgo a la persona y sus futuras generaciones.

Conflicto de intereses: el autor declara no tener ningún conflicto de intereses.

\section{Bibliografía}

Act now on CRISPR babies. (2019). Nature, 570(7760), 137-137. doi:10.1038/d41586019-01786-3.

Bermeo-Antury, E., \& Quimbaya, M. (2016). Secuenciación De Próxima Generación Y Su Contexto Eugenésico En El Embrión Humano. Persona Y Bioética, 20(2), 205231.

Bernal, C. A. (2016). Metodologia de la Investigación (3 ed.). Bogotá: Pearson Educación

Centro Cochrane Iberoamericano, traductores. Manual Cochrane de Revisiones Sistemáticas de Intervenciones, versión 5.1.0 [actualizada en marzo de 2011] [Internet]. Barcelona: Centro Cochrane Iberoamericano; 2012. Disponible en http://www.cochrane.es/?q=es/node/269

Collins, F. S., M.D., Ph.D. (2018, November 28). Statement on Claim of First Gene-Edited Babies by Chinese Researcher. Retrieved from https://www.nih.gov/about-nih/whowe-are/nih-director/statements/statementclaim-first-gene-edited-babies-chineseresearcher

Cong, L., et al. (2013). Multiplex Genome Engineering Using CRISPR/Cas Systems. Science, 339 (6121), 819-823.

C. (1997). Convention for the protection of human rights and dignity of the human being with regard to the application of biology and medicine: Convention on human rights and biomedicine: Oviedo, 4. IV. European Treaty Series - No. 164). Strasbourg: Council of Europe.

Cribbs, A. P., \& Perera, S. (2017). Science and Bioethics of CRISPR-Cas9 Gene Editing: An Analysis Towards Separating Facts and Fiction. The Yale journal of biology and medicine, 90(4), 625-634.

Cyranoski, D., \& Reardon, S. (2015). Embryo editing sparks epic debate. Nature, 520(7549), 593-594.

Dance, A. (2017). Better beings? Nature Biotechnology, 35(11), 1006-1011

De Lecuona, I., Casado, M., Marfany, G., Lopez Baroni, M., \& Escarrabill, M. (2017). Gene Editing in Humans: Towards a Global and Inclusive Debate for Responsible Research. YALE JOURNAL OF BIOLOGY AND MEDICINE, 90, 673-681.

Doudna, J. A., \& Charpentier, E. (2014). The new frontier of genome engineering with CRISPR-Cas9. Science, 346(6213), 12580961-1258096-9

Gamboa-Bernal, G. A. (2016). La Edición De Genes A Estudio: Los Problemas Bioéticos 
Que Puede Tener Esta Nueva Tecnología. Persona Y Bioética, 20(2), 125-131

Gómez-Tatay, L., \& Aznar, J. (2019). CRISPRCAS9. El mayor avance en técnicas de edición genética requiere una reflexión ética. Cuadernos De Bioética, 30(99), 171185.

Krimsky, S. (2019). Ten ways in which He Jiankui violated ethics. Nature Biotechnology, 37(1), 19-20.

Kyrou, K., Hammond, A. M., Galizi, R., Kranjc, N., Burt, A., Beaghton, A. K., Crisanti, A. (2018). A CRISPR-Cas9 gene drive targeting doublesex causes complete population suppression in caged Anopheles gambiae mosquitoes. Nature Biotechnology, 36(11), 1062-1066

Ledford, H. (2016). Riding the CRISPR wave. Nature, 531, 156-159

Ledford, H. (2019). CRISPR babies: When will the world be ready? Nature, 570(7761), 293296

Li, J., Walker, S., Nie, J., \& Zhang, X. (2019). Experiments that led to the first geneedited babies: The ethical failings and the urgent need for better governance. Journal of Zhejiang University-SCIENCE B, 20(1), 32-38

Normile, D. (2018). CRISPR bombshell: Chinese researcher claims to have created geneedited twins. Science
Riordan, S. M., Heruth, D. P., Zhang, L. Q., \& Ye, S. Q. (2015). Application of CRISPR/Cas9 for biomedical discoveries. Cell \& Bioscience, 5(1), 1-11

Hernandez Sampieri, R. (2014). Metodología de la investigación. (6 ed.). Mexico: McGrawHill

Sgreccia, E. (2014). Manual de bioética. Madrid: Biblioteca de Autores Cristianos

Uchiyama, M., Nagai, A., \& Muto, K. (2018). Survey on the perception of germline genome editing among the general public in Japan. Journal of Human Genetics, 63(6), 745-748.

Vogel, G. (2015). Research on gene editing in embryos is justified, group says. Science

Vogel, G. (2015). Embryo engineering alarm. Researchers call for restraint in genome editing. Science, 347(6228), 1301-1301 